Supporting Information

\title{
Activation energy of hydrogen
}

\author{
adsorption on $\operatorname{Pt(111)~in~alkaline~}$
}

media: an impedance spectroscopy

\section{study at variable temperatures}

Luis E. Botello, Juan M. Feliu and Victor Climent*

Instituto Universitario de Electroquímica, Universidad de Alicante, Carretera San

Vicente del Raspeig s/n, E-03690 San Vicente del Raspeig, Alicante, Spain

*E-mail: victor.climent@ua.es 


\section{Relationship between charge transfer resistance and the standard rate constant}

The hydrogen adsorption reaction that takes place in the platinum surface on alkaline media is described by equation (1):

$$
\mathrm{H}_{2} \mathrm{O}+e^{-} \rightleftharpoons \mathrm{H}_{\text {ads }}+\mathrm{OH}^{-}
$$

Which means that the rate of the reaction is given by the Butler-Volmer type relation assuming a Frumkin adsorption isotherm:

$$
v=\frac{d \theta}{d t}=k^{0}(1-\theta) \exp \left[-\beta f\left(E-E_{a}^{0}\right)-\alpha g \theta\right]-k^{0} \theta \frac{c_{O H^{-}}}{c^{0}} \exp \left[(1-\beta) f\left(E-E_{a}^{0}\right)+(1-\alpha) g \theta\right]
$$

Where $v$ is the reaction rate, $\theta$ is the surface coverage coefficient, $t$ is time, $k^{0}$ is the standard rate constant of the reaction, $f=\frac{F}{R T}, \beta$ is the charge transfer coefficient, $c^{0}$ is the standard state concentration, $E$ is the electrode potential, $E_{a}^{0}$ is the adsorption standard potential, $\alpha$ is the symmetry coefficient of the adsorption reaction, $g$ is the adsorbed hydrogen interaction factor and $\mathrm{C}_{\mathrm{OH}^{-}}$is the bulk hydroxyl concentration. The reaction rate is $v=0$ at the equilibrium potential, which results in the following relations:

$$
\begin{gathered}
v^{0}=k^{0}(1-\theta) \exp \left[-\beta f\left(E_{e q}-E_{a}^{0}\right)-\alpha g \theta\right]=k^{0} \theta \frac{c_{O H^{-}}}{c^{0}} \exp \left[(1-\beta) f\left(E_{e q}-E_{a}^{0}\right)+(1-\alpha) g \theta\right] \\
k^{0}(1-\theta) \exp [-\alpha g \theta]=\frac{v^{0}}{\exp \left[-\beta f\left(E_{e q}-E_{a}^{0}\right)\right]} \\
k^{0} \theta \frac{c_{O H^{-}}}{c^{0}} \exp [(1-\alpha) g \theta]=\frac{v^{0}}{\exp \left[(1-\beta) f\left(E_{e q}-E_{a}^{0}\right)\right]}
\end{gathered}
$$

After substitution of equations (4) and (5) into equation (2):

$$
v=v^{0}\{\exp [-\beta f \eta]-\exp [(1-\beta) f \eta]\}
$$

Where $\eta=E-E_{e q}$ is the overpotential. An expression for $v^{0}$ can be found dividing equation (5) by equation (4):

$$
\frac{c_{O H^{-}}}{c^{0}} \frac{\theta}{1-\theta} \exp (g \theta)=\exp \left[-f\left(E_{e q}-E_{a}^{0}\right)\right]
$$

Equation (7) is then substituted into equation (4), resulting in:

$$
v^{0}=k^{0}(1-\theta)^{(1-\beta)}\left(\frac{c_{O H^{-}}}{c^{0}}\right)^{\beta} \theta^{\beta} \exp [g \theta(\beta-\alpha)]
$$

Equation (8) depends only on the surface coverage, which can be controlled by the applied potential. A direct current approach to find the relation between the charge 
transfer resistance and the standard rate constant can be found by obtaining the Taylor series expansion of equation (6):

$$
v=-v^{0} f \eta
$$

The current density of adsorption in a $\mathrm{Pt}(111)$ electrode is given by:

$$
j=-q_{M L} \frac{d \theta}{d t}=q_{M L} v^{0} f \eta
$$

Where $q_{M L}$ is the surface charge for a $\operatorname{Pt}(111)$ basal plane. Then, by applying Ohm's law for small deviations from the equilibrium potential, one obtains:

$$
R_{c t}=\frac{\eta}{j}=\frac{1}{q_{M L} v^{0} f}=\frac{1}{q_{M L} f k^{0}(1-\theta)^{(1-\beta)}\left(\frac{c_{O H^{-}}}{c^{0}}\right)^{\beta} \theta^{\beta} \exp [g \theta(\beta-\alpha)]}
$$

Which ends in a simpler form in the common case where $\beta \approx \alpha \approx 0.5$. The potential dependent surface coverage can be directly evaluated from cyclic voltammetry measurements and then the standard rate constant can be obtained by fitting the dependence of the charge transfer resistance with the applied potential.

This kinetic treatment is not the only way to arrive to equation (11), it can also be obtained from a frequency treatment:

$$
\begin{aligned}
j & =j_{s}+\bar{j} e^{i \omega t} \\
E & =E_{s}+\bar{E} e^{i \omega t} \\
\theta & =\theta_{s}+\bar{\theta} e^{i \omega t}
\end{aligned}
$$

Where $X_{S}$ are the stationary components of the parameters and $\bar{X}$ are the phasors. The current variation is obtained from equation (12), which is written according to the chain rule:

$$
\partial j=\left(\frac{\partial j}{\partial E}\right)_{\theta} \partial E+\left(\frac{\partial j}{\partial \theta}\right)_{E} \partial \theta=j_{E} \partial E+j_{\theta} \partial \theta
$$

Which means:

$$
\begin{gathered}
\bar{j} e^{i \omega t}=j_{E} \bar{E} e^{i \omega t}+j_{\theta} \bar{\theta} e^{i \omega t} \\
\bar{j}=j_{E} \bar{E}+j_{\theta} \bar{\theta}
\end{gathered}
$$

Then, the change in current density can be written as: 


$$
\begin{gathered}
\bar{j} e^{i \omega t}=-q_{M L} \frac{d\left(\bar{\theta} e^{i \omega t}\right)}{d t}=-q_{M L} \bar{\theta} e^{i \omega t} i \omega \\
\bar{\theta}=\frac{\bar{j}}{-q_{M L} i \omega}
\end{gathered}
$$

Equation (17b) can then be substituted into equation (16b) and the ratio of the phasors for the potential and the current density is the definition of the impedance:

$$
\hat{Z}=\frac{\bar{E}}{\bar{j}}=\frac{1}{j_{E}}+\frac{j_{\theta}}{j_{E} q_{M L}} \frac{1}{i \omega}
$$

This can be intuitively viewed as the impedance of the adsorption process, which has a charge transfer resistance and an associated capacitive behavior, but an expression of $1 / j_{E}$ still needs to be found. The differential form of $j_{E}$ as seen in equation (15) means that it can be described with a Butler-Volmer equation, recalling equation (6):

$$
j=-q_{M L} v^{0}\{\exp (-\beta f \eta)-\exp [(1-\beta) f \eta]\}
$$

Then, taking the derivative of equation (19):

$$
j_{E}=\left(\frac{\partial j}{\partial E}\right)_{\theta}=-q_{M L} \nu^{0}(\theta)\{-\beta f \exp (-\beta f \eta)-(1-\beta) f \exp [(1-\beta) f \eta]\}
$$

At the equilibrium potential, $\eta=0$ and equation (20) becomes:

$$
j_{E}=q_{M L} f v^{0}(\theta)
$$

Then, equation (20) represents the charge transfer resistance of the adsorption process:

$$
\frac{1}{j_{E}}=\frac{1}{q_{M L} f v^{0}(\theta)}=\frac{1}{q_{M L} f k^{0}(1-\theta)^{(1-\beta)}\left(\frac{\left.c_{O H^{-}}\right)^{\beta}}{c^{0}} \theta^{\beta} \exp [g \theta(\beta-\alpha)]\right.}
$$

Which is identical to equation (11) and further proves the applicability of the $R_{c t}$ equation obtained to establish the relation between the charge transfer resistance and the standard rate constant.

\section{Equivalent electrical circuit equation}

The impedance $\hat{Z}$ of the equivalent circuit depends on the frequency according to:

$$
\hat{Z}=R_{s}+\frac{1}{Q(i \omega)^{\lambda}+\frac{1}{R_{c t}+\frac{1}{i \omega C_{a d}}}}
$$


Where $\omega$ is the frequency, $i=\sqrt{-1}$, and the impedance of the double layer is expressed as a constant phase element to account for the dispersion of capacitance at the electrode, with $Q$ as a parameter related to the electrode capacitance and $\lambda$ is the constant phase exponent.

\section{Apparent Arrhenius tendency}

The $R_{c t}$ data obtained from the fittings was then plotted against the applied potential and the logarithm of the same value was plotted with respect to the inverse of the temperature to initially check for an Arrhenius type behavior. The results are shown in figure S1.
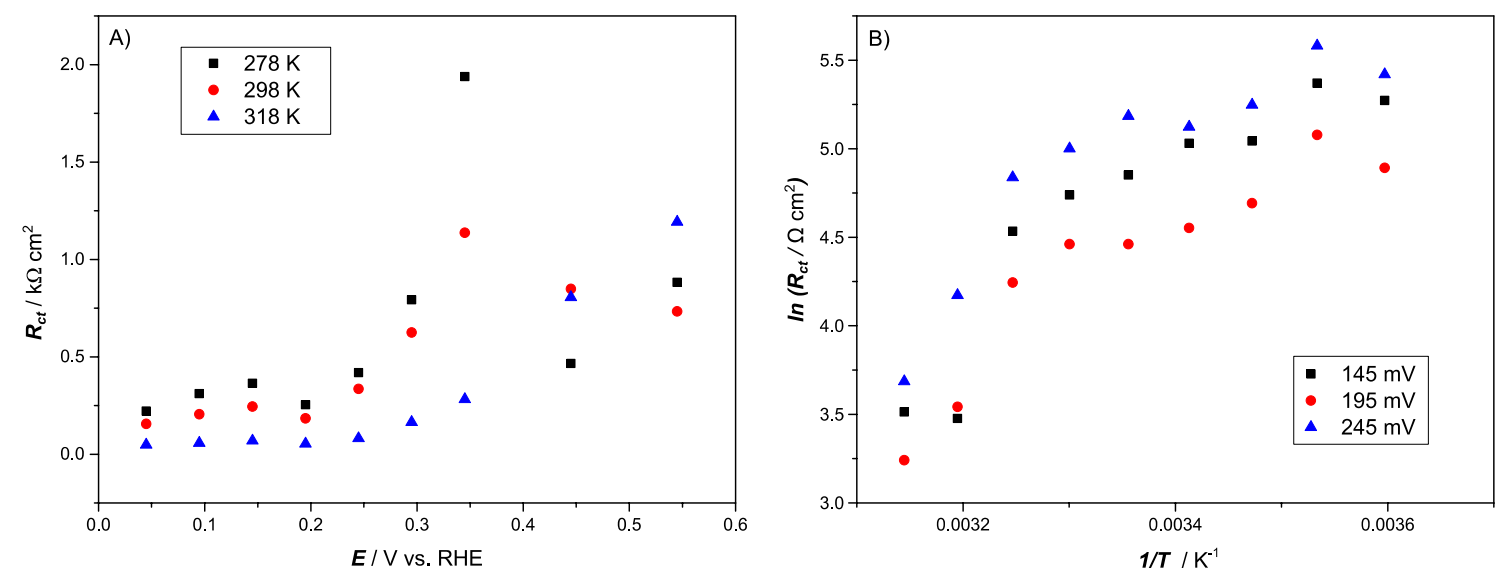

Figure S1. Charge transfer resistance with respect to the applied potential (A) and natural logarithm of the charge transfer resistance against the inverse of the temperature for three representative potential (B).

\section{Datasets tables}

The values for the equivalent circuit elements obtained from nonlinear fitting are presented in the following tables. The first table is from fits obtained at a fixed potential with different temperatures. The next ones were obtained at different temperatures with different applied potentials, here are represented at three key temperatures to make the change of values clear. 
Table S1. Values of circuit elements obtained at $5{ }^{\circ} \mathrm{C}$ from different applied potentials.

\begin{tabular}{cccccc}
\hline $\mathrm{E}(\mathrm{mV})$ & $\begin{array}{c}\mathrm{R}_{\mathrm{s}} \\
\left(\Omega \mathrm{cm}^{2}\right)\end{array}$ & $\begin{array}{c}\mathrm{R}_{\mathrm{ct}} \\
\left(\Omega \mathrm{cm}^{2}\right)\end{array}$ & $\begin{array}{c}\mathrm{C}_{\mathrm{dl}} \\
\left.(\mu \mathrm{F} \mathrm{cm})^{-2}\right)\end{array}$ & $\begin{array}{c}\mathrm{C}_{\mathrm{ad}} \\
\left(\mathrm{mF} \mathrm{cm}^{-2}\right)\end{array}$ & $\lambda \pm 0.01$ \\
\hline 45 & $25 \pm 5$ & $116 \pm 23$ & $23 \pm 2$ & $0.49 \pm 0.02$ & 0.97 \\
95 & $25 \pm 6$ & $162 \pm 32$ & $21 \pm 1$ & $0.52 \pm 0.03$ & 0.99 \\
145 & $24 \pm 6$ & $190 \pm 42$ & $21 \pm 1$ & $0.54 \pm 0.03$ & 0.99 \\
195 & $25 \pm 6$ & $133 \pm 70$ & $24 \pm 2$ & $0.51 \pm 0.02$ & 0.98 \\
245 & $25 \pm 2$ & $219 \pm 118$ & $26 \pm 1$ & $0.39 \pm 0.02$ & 0.98 \\
305 & $25 \pm 2$ & $414 \pm 220$ & $30 \pm 1$ & $0.21 \pm 0.02$ & 0.96 \\
345 & $26 \pm 6$ & $1013 \pm 587$ & $25 \pm 1$ & $0.05 \pm 0.01$ & 0.98 \\
445 & $26 \pm 6$ & $243 \pm 78$ & $29 \pm 3$ & $4.79 \times 10^{-5}$ & 0.96 \\
545 & $26 \pm 6$ & $461 \pm 600$ & $28 \pm 4$ & $2.78 \times 10^{-4}$ & 0.97 \\
\hline
\end{tabular}

Table S2. Values of circuit elements obtained at $25^{\circ} \mathrm{C}$ from different applied potentials.

\begin{tabular}{cccccc}
\hline E (mV) & $\begin{array}{c}\mathrm{R}_{\mathrm{s}} \\
\left(\Omega \mathrm{cm}^{2}\right)\end{array}$ & $\begin{array}{c}\mathrm{R}_{\mathrm{ct}} \\
\left(\Omega \mathrm{cm}^{2}\right)\end{array}$ & $\begin{array}{c}\mathrm{C}_{\mathrm{dl}} \\
\left(\mu \mathrm{F} \mathrm{cm}^{-2}\right)\end{array}$ & $\begin{array}{c}\mathrm{C}_{\mathrm{ad}} \\
\left.(\mathrm{mF} \mathrm{cm})^{-2}\right)\end{array}$ & $\lambda \pm 0.01$ \\
\hline 45 & $16.9 \pm 0.3$ & $81 \pm 10$ & $22 \pm 1$ & $0.50 \pm 0.02$ & 0.98 \\
95 & $17.1 \pm 0.4$ & $107 \pm 14$ & $21 \pm 1$ & $0.53 \pm 0.02$ & 0.99 \\
145 & $17.3 \pm 0.5$ & $128 \pm 16$ & $22 \pm 1$ & $0.54 \pm 0.01$ & 0.99 \\
195 & $17.8 \pm 0.7$ & $96 \pm 23$ & $25 \pm 2$ & $0.51 \pm 0.03$ & 0.98 \\
245 & $16 \pm 3$ & $175 \pm 34$ & $36 \pm 2$ & $0.41 \pm 0.02$ & 0.94 \\
305 & $18 \pm 1$ & $326 \pm 96$ & $25 \pm 2$ & $0.20 \pm 0.01$ & 0.98 \\
345 & $19 \pm 1$ & $594 \pm 227$ & $25 \pm 2$ & $0.05 \pm 0.01$ & 0.99 \\
445 & $19 \pm 1$ & $443 \pm 151$ & $30 \pm 5$ & $9.11 \times 10^{-4}$ & 0.96 \\
545 & $19 \pm 2$ & $383 \pm 154$ & $30 \pm 6$ & $5.29 \times 10^{-4}$ & 0.96 \\
\hline
\end{tabular}


Table S3. Values of circuit elements obtained at $45^{\circ} \mathrm{C}$ from different applied potentials.

\begin{tabular}{cccccc}
\hline E (mV) & $\begin{array}{c}\mathrm{R}_{\mathrm{s}} \\
\left(\Omega \mathrm{cm}^{2}\right)\end{array}$ & $\begin{array}{c}\mathrm{R}_{\mathrm{ct}} \\
\left(\Omega \mathrm{cm}^{2}\right)\end{array}$ & $\begin{array}{c}\mathrm{Cdl} \\
\left(\mu \mathrm{F} \mathrm{cm}{ }^{-2}\right)\end{array}$ & $\begin{array}{c}\mathrm{Cad}_{\mathrm{ad}} \\
\left(\mathrm{mF} \mathrm{cm}^{-2}\right)\end{array}$ & $\lambda \pm 0.01$ \\
\hline 45 & $12.3 \pm 0.3$ & $25 \pm 1$ & $25 \pm 1$ & $0.53 \pm 0.06$ & 0.98 \\
\hline 95 & $12.7 \pm 0.4$ & $30 \pm 3$ & $22 \pm 1$ & $0.55 \pm 0.05$ & 0.99 \\
\hline 145 & $13.0 \pm 0.4$ & $36 \pm 3$ & $24 \pm 1$ & $0.55 \pm 0.01$ & 0.99 \\
\hline 195 & $13.2 \pm 1.5$ & $28 \pm 6$ & $29 \pm 2$ & $0.52 \pm 0.06$ & 0.97 \\
\hline 245 & $13.2 \pm 1.6$ & $42 \pm 10$ & $31 \pm 1$ & $0.45 \pm 0.01$ & 0.97 \\
\hline 305 & $14.2 \pm 2.0$ & $86 \pm 15$ & $30 \pm 1$ & $0.20 \pm 0.01$ & 0.97 \\
\hline 345 & $14.4 \pm 3.6$ & $147 \pm 84$ & $28 \pm 2$ & $0.05 \pm 0.02$ & 0.98 \\
\hline 445 & $12.1 \pm 0.8$ & $421 \pm 226$ & $34 \pm 1$ & $6.40 \times 10^{-4}$ & 0.97 \\
\hline 545 & $12.4 \pm 0.9$ & $623 \pm 383$ & $26 \pm 1$ & $1.19 \times 10^{-3}$ & 0.99 \\
\hline
\end{tabular}

